\title{
Suppressive interactions between moving patterns: Role of velocity
}

\author{
ROBERT J. SNOWDEN \\ University of Cambridge, Cambridge, England
}

\begin{abstract}
Detection and use of motion in complex environments requires movement measurements to be combined. The role of velocity in the suppressive interaction between patterns that move in orthogonal directions was assessed. When $d_{\max }$ was measured, it was found that a range of medium to high speeds of vertical motion all had a suppressive effect upon detecting horizontal motion. When $d_{\min }$ was measured, only a range of low velocities caused a disruption of performance. Thus, whilst velocity is an important parameter in determining the size of the suppressive effect, it has nonparallel influences at the upper and lower end of the displacement range.
\end{abstract}

The Gestalt psychologists recognized that objects may be grouped according to rules of similarity and proximity. One such rule involved "common fate" (Wertheimer, 1923/1958): Objects with similar movement (common fate) appear to group together. A more recent demonstration of such a phenomenon is that moving areas within a random-dot pattern can be segregated purely on the basis of their different movements (e.g., see Braddick, 1974). It is clear that, to achieve this, the visual system must combine movement information distributed across the space of the surface. This is, however, quite a challenge, as each stationary frame provides no clues as to the areas whose motions are different. Only by means of motion information are these different areas perceived to exist. The visual system is therefore faced with the problem of trying to combine motion information across a surface, while avoiding combining motion information that belongs to a different surface. Since it is expected that the motion will vary only gradually within a surface, whereas across a surface boundary it will vary rapidly, one possible solution might be to combine motion information that is "similar" both in terms of its location (i.e., the information is obtained from nearby points in the image) and velocity (both speed and direction), while not combining that which is "different."

The situation is even more complicated when transparent surfaces are considered. If two moving random-dot patterns are superimposed, they may appear to flow through one another (e.g., see Clarke, 1977; Siegel \& Andersen, 1988; van Doorn \& Koenderink, 1982b). Indeed Andersen (1989) provides evidence that subjects can

\footnotetext{
I thank O. J. Braddick for supervising this work. My appreciation also goes to two anonymous referees whose criticisms greatly improved the manuscript. I also thank the Science and Engineering Research Council of Great Britain for financial support. Requests for reprints should be sent to Robert J. Snowden, Department of Brain and Cognitive Sciences, E25-236, Massachusetts Institute of Technology, Cambridge, MA 02139 .
}

accurately segregate up to three superimposed texture patterns that have different rates of movement. Although the information that specifies each surface overlaps the information specifying the other surfaces completely in space, the visual system is still able to resolve these surfaces (though there are limits; see Andersen, 1989; van Doorn \& Koenderink, 1982c). Such a feat appears effortless, yet consideration of the necessary computation soon reveals the complexity of the required calculations. Indeed current computational models of coherent motion do not account for transparency phenomena (e.g., Horn \& Shunck, 1981; Wang, Mathur, \& Koch, 1989; Yuille \& Grzywacz, 1988), for they tend to "smooth" the velocity field and thus provide a single-surface answer by definition. Indeed, in the case of transparent surfaces moving in opposite directions, the smoothed surface would have zero velocity.

Recently I have examined the possibility that transparent motions are not completely independent of one another, or, in other words, that they might exhibit interactions (Snowden, 1989). Two superimposed randomdot patterns moving in orthogonal directions were shown to have mutually suppressive effects. This was demonstrated by measuring $d_{\max }$ for horizontal motions (the largest displacement to which direction can be reliably assigned; see Braddick, 1980) in the presence and absence of vertical movement of another pattern. Performance was shown to be severely affected by the orthogonal movement. This suggests that motions of different directions might have a suppressive influence on one another. The present study was designed to elaborate this notion further. I measured how the rate of the vertical motion (termed the background movement) would affect the threshold $d_{\max }$, and made similar measurements using a measure of the minimum motion threshold ( $d_{\text {min }}$ : the smallest displacement to which direction can be reliably assigned). The results show that although the speed of the background motion is important in determining the size of the suppressive effect, it does so in a nonparallel manner for the different threshold measures. 


\section{GENERAL METHOD}

\section{Stimuli}

Random-dot patterns were produced by software run upon a PDP11/10 minicomputer, which controlled the output of a Sigma QVEC Vector Generator to a Hewlett-Packard 1319 CRT display (P31 phosphor). The software was designed upon the following principles: All points in one pattern were drawn relative to a starting point via an increment procedure, the increment value being chosen randomly from within a set range from point to point. The increment function automatically "wraps around" to the opposite side of the pattern any point that falls beyond the outer edge of the display area. The positioning of the point in the other axis was chosen from a list of random coordinates housed in the memory of the computer. Four hundred points were calculated and displayed per pattern. Displacements of all these points along one axis could be achieved by simply reassigning the starting point. Due to the wrapping around, the reassigning of the starting point had the effect of a smooth motion within a window. The test pattern was always written, via the increment procedure, along the $x$ axis, so its displacement was always horizontal. The background pattern was always written, via the increment function, along the $y$ axis, so its displacements were always vertical. The two patterns were superimposed within each single frame on the screen, so 800 points were visible. Of course, when both patterns were stationary, it was impossible to tell if one particular dot belonged to the test or to the background pattern.

The background stimulus consisted of a sequence in which the pattern was first stationary, then underwent vertical motion, and then was stationary once more. The stationary components were both of 100 -msec duration, whereas the motion phase lasted $200 \mathrm{msec}$. The screen refresh rate was $100 \mathrm{~Hz}$, so the motion phase normally consisted of displacements every $10 \mathrm{msec}$. The displacements could be of $1,2,4,8,16,32$, or 64 pixels, which produced velocities of $0.4^{\circ}-25.6^{\circ}$ per sec. Lower background velocities were produced by displacing the pattern by 1 pixel every 2 or 4 frames $\left(0.2^{\circ}\right.$ or $0.1^{\circ}$ per sec), and an additional condition was run in which no displacement occurred. These 10 conditions gave a range of background velocities of $0^{\circ}-25.6^{\circ}$ per sec. The test pattern was stationary during the whole of the background pattern's duration, apart from one discrete horizontal displacement at the midpoint of the motion phase of the background. The amplitude of this displacement was the dependent variable.

The display area subtended $3.7^{\circ}$ from the viewing distance of $3 \mathrm{~m}$. Each point was a brightly illuminated dot with a diameter of $0.5 \mathrm{~mm}$, and the displays were viewed in a dimly lit room. These conditions ensured that there was no visible phosphor trace.

\section{Procedures}

In both experiments, the subjects were told of the expected appearance of the stimulus. They were told to ignore the vertical motion (for which up or down directions were randomized from trial to trial), and to report the perceived direction of the horizontal motion (left or right directions were randomized) by pressing one of two buttons. No feedback was given. Prior to each trial, a 0.5 -sec fixation point, centered with respect to the presentation of the forthcoming stimulus, appeared immediately before onset.

Thresholds were gathered by different procedures in each experiment. In Experiment 1, a staircase procedure was employed, which consisted of incrementing the amplitude of test-pattern displacement if the subject correctly identified the direction of motion on two successive occasions within a single staircase, and decrementing the amplitude following each incorrect response. Such a procedure tracks the $71 \%$ correct threshold. Two or more correct responses followed by one incorrect response were termed a reversal. Each staircase terminated after 11 such reversals. The staircases commenced with a displacement amplitude of $21.7^{\prime}$; they were incremented/decremented by $20 \%$ steps before the first reversal, by
$10 \%$ steps between the first and second reversal, and by $5 \%$ steps thereafter. All amplitudes of displacement after the third reversal were stored, and their mean was taken as the threshold value $d_{\max }$. In Experiment 2, the method of constant stimuli was employed. After pilot trials, in which estimates of $d_{\text {min }}$ were obtained, a range of displacements was chosen for presentation. This range was drawn from displacements from 1 to 15 pixels $(13-200 \mathrm{sec})$. Six displacements were selected and presented 30 times each for the 10 conditions, which totalled 1,800 trials per subject. Because this was considered excessive to run in one block, the trials were split into two experimental sessions, each subject making 15 of the 30 responses per displacement in each session. The blocks were combined, and error versus displacement graphs were plotted for each of the 10 conditions. Linear interpolation was applied between points, and the displacement at which the function crossed the $25 \%$ error mark was termed $d_{\min }$. If, due to "kinks" in the function, this point was crossed more than once, the average was taken between crossings. This only occurred for four of the thirty points to be reported.

\section{Subjects}

A total of 4 subjects participated in the course of these experiments, 2 in both experiments and 2 in one experiment each. All had normal or corrected-to-normal vision and were experienced psychophysical observers. All but R.S. were naive as to the aims of the experiments. All viewing was binocular.

\section{RESULTS}

\section{Experiment 1}

In this experiment, I determined how the velocity of the background pattern affects the threshold $d_{\max }$ for the test pattern. Figure 1 plots $d_{\max }$ as a function of the background velocity for 3 subjects. With a stationary background, $d_{\max }$ is around $22^{\prime}$, in accord with previous estimates under similar conditions (Snowden, 1989). As the velocity of the background is increased, $d_{\max }$ appears to undergo no systematic change until the velocity is around $0.6^{\circ}-1.0^{\circ}$ per sec. Around this velocity, $d_{\max }$ falls sharply to a level of around 8-12 $\mathrm{min}$, with a minimum occurring for background velocities around $3^{\circ}$ per sec. At the greatest velocity employed, $d_{\max }$ is somewhat higher, suggesting that at still greater velocities performance might continue improving.

\section{Experiment 2}

In this experiment, I determined how the velocity of the background pattern affects the threshold $d_{\text {min }}$ for the test pattern. Figure 2 plots $d_{\min }$ as a function of the velocity of the background pattern. (Note that the ordinate is now in seconds. Furthermore, the ordinate has been inverted so that "better performance" corresponds to points at the top of the graph, hence facilitating comparisons with the $d_{\max }$ data.) When the background is stationary, $d_{\min }$ varies between 55 and $95 \mathrm{sec}$. Such large variations between subjects when measuring $d_{\text {min }}$ has been previously noted (Baker \& Braddick, 1985a); at the equivalent field size, for example, they report $d_{\text {min }}$ of $30-100 \mathrm{sec}$. As the velocity of the background pattern is increased, $d_{\min }$ rises (hence the functions fall), so that for a background velocity of $0.4^{\circ}$ per sec, $d_{\text {min }}$ is almost double the value obtained with a stationary background. 


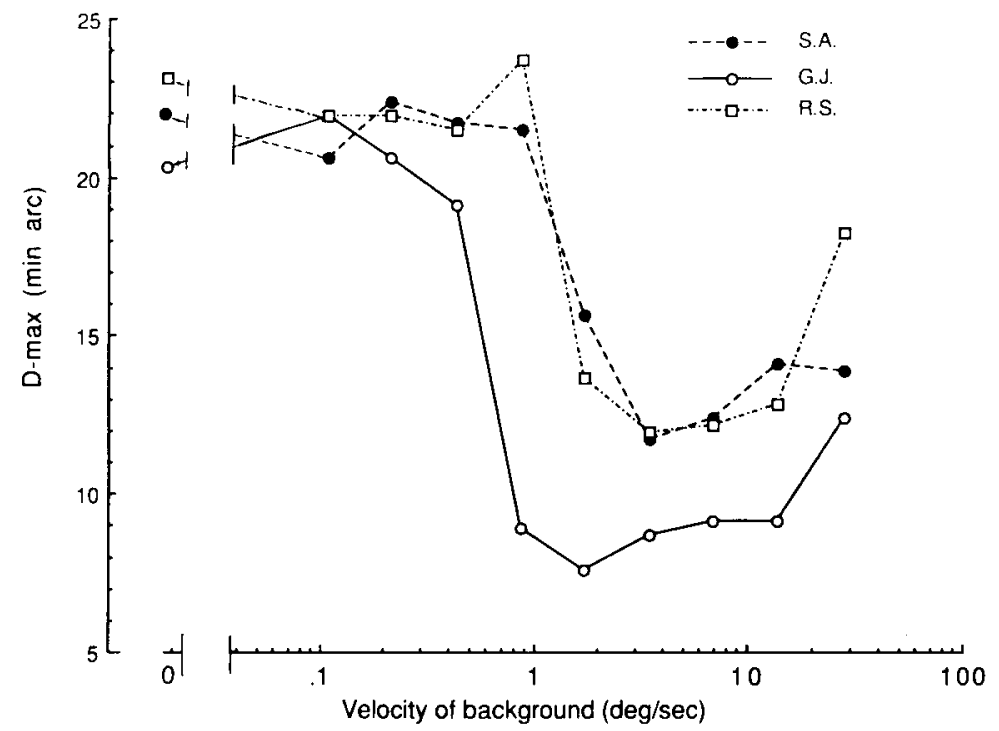

Figure 1. The threshold $d_{\max }$ for the horizontal test motion is plotted against the velocity of the superimposed background pattern. Fach function represents data from 1 subject. Thresholds for a stationary background are around $23 \mathrm{~min}$ and remain so as the velocity of the background pattern increases to around $0.6^{\circ}$ per sec. When the velocity of the background pattern is $1-10^{\circ}$ per sec, $d_{\max }$ is considerably reduced. Finally, at the highest velocity of the background employed in this study, there is an upturn in the value $d_{\max }$.

As the velocity of the background pattern is increased further, $d_{\min }$ falls sharply (performance improves) to a value of around $50 \mathrm{sec}$ (for all subjects!), and it remains at this level even when the velocity of the background is increased even more. It is perhaps worth noting that, for all subjects, this level of $50 \mathrm{sec}$ is somewhat lower than when the background was stationary.

\section{DISCUSSION}

The present results help delineate the bounds of the suppressive interactions between motions of orthogonal direction. When $d_{\max }$ was measured, performance was relatively unaffected by low background velocities up to around $1^{\circ}$ per sec. At higher background velocities, per-

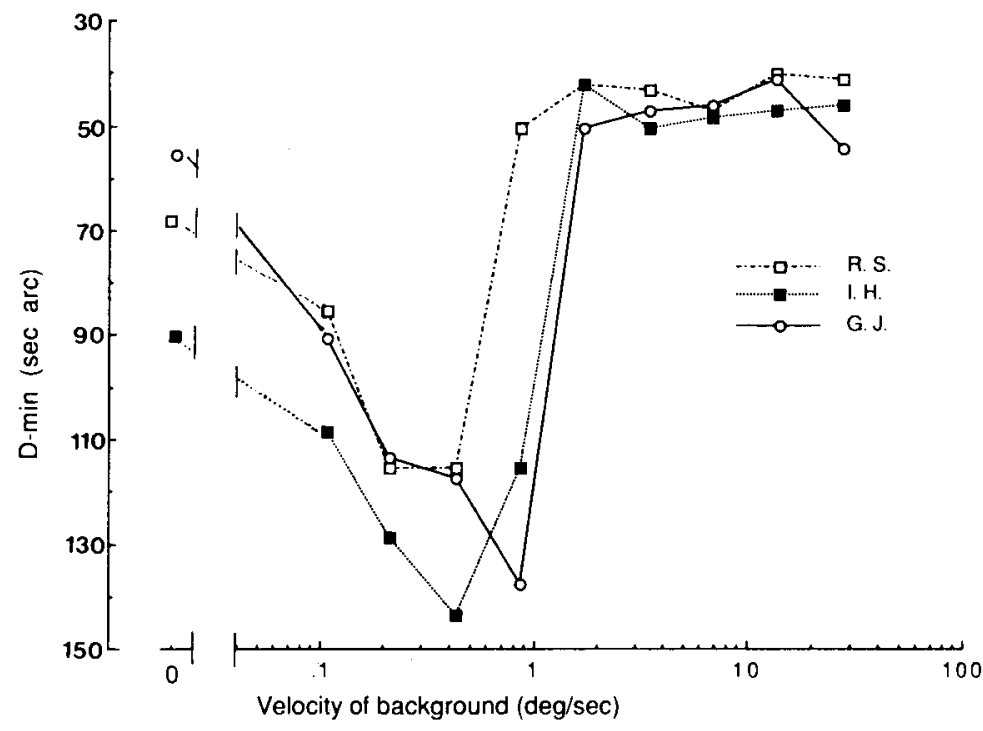

Figure 2. The threshold $d_{\min }$ for the horizontal test motion is plotted against the velocity of the superimposed background pattern. All conditions are as in Figure 1. However, the ordinate is now in seconds, and it is plotted with $d_{\min }$ decreasing up the ordinate. This aids in comparing the data from $d_{\max }$, since better performance is indicated on both graphs by points nearer to the top. 
formance showed a marked decrease, with a shallow minimum occurring for velocities around $3^{\circ}$ per sec. At the highest background velocity tested, there is an indication that performance is somewhat better than at slightly lower velocities. How can such results be interpreted? One popular notion is that motion is signaled by bilocal motion detectors (e.g., Barlow \& Levick, 1965; Reichardt, 1961; van Doorn \& Koenderink, 1982c). Such detectors are "velocity-tuned" by the ratio of their characteristic spans and delays, and $d_{\max }$ could therefore be related to the detectors with the largest spans that are sufficiently excited by the displays. Motions that also excite these spans might therefore be responsible for the suppressive effect. Surprisingly, performance was not maximally disrupted by the highest background velocities but by a somewhat lower range. In quantitative terms, $d_{\max }$ (which would have been approximately $23^{\prime}$ ) was maximally effected at velocities around $3^{\circ}$ per sec or $1.7^{\prime}$ displacement per frame. Indeed, according to the equations of van Doorn and Koenderink (1982a) speeds of $3^{\circ}$ per sec are subserved by units with spans centered on $8^{\prime}$. Clearly, then, the suppressive effect of orthogonal motion is not restricted to motions of similar speeds or displacements; instead, a broad range of medium to high velocities may affect performance when gauged by the threshold $d_{\max }$.

The small improvement in performance at the very highest velocities used is also of interest. This might well be due to the background pattern's reaching its own " $d_{\text {max. }}$." Displacement per frame was 14 ' at this velocity, which might be near threshold when the reduction in $d_{\max }$ due to short frame duration is taken into account (Baker \& Braddick, 1985b). Displacements near $d_{\max }$ have already been shown to have only a small suppressive effect (Snowden, 1989); hence the improvement in performance at the highest background velocity tested.
The results relating to the threshold $d_{\min }$ can be considered in an analogous way; $d_{\min }$ might be signaled by the bilocal detector with the smallest span that is sufficiently stimulated. Once again, we might expect the maximum suppression when the background and test motions are of similar speeds or displacements. But this is not the case. Poorest performance occurs when the background velocity is $0.4^{\circ}$ per sec, which would be served by units with spans around $2.4^{\prime}$ according to the equations of van Doorn and Koenderink (1982a). Thus $d_{\min }$ can be affected by a broad range of slow movements in an orthogonal direction. So, although each of the two thresholds measured can be disrupted by movements in an orthogonal direction, and although both exhibit a dependency of this effect upon the velocity of the movement, the range of velocities over which each is affected is different. Indeed, as can be gathered from Figure 3, the background velocity that maximally suppresses $d_{\max }$ has a minimal effect upon $d_{\min }$, and vice versa.

Comparison of the present data with previous research is difficult, because little work has been done to assess interactions between motions. Van Doorn and Koenderink (1982b), however, provide some evidence. When two moving patterns are rapidly and alternatively sampled in time, transparency is seen. Under these conditions, thresholds for detecting any coherent motion are lower when the two motions have the same direction than when they have different directions (opposite or orthogonal). This is true for a range of speeds, but because both motions in van Doorn and Koenderink's study had the same speed, it is hard to relate their study's results to the present ones. Marshak and Sekuler (1979) and Mather and Moulden (1980) have both demonstrated that motions with dissimilar directions exert an influence upon one another, such that they appear to move in directions that are more

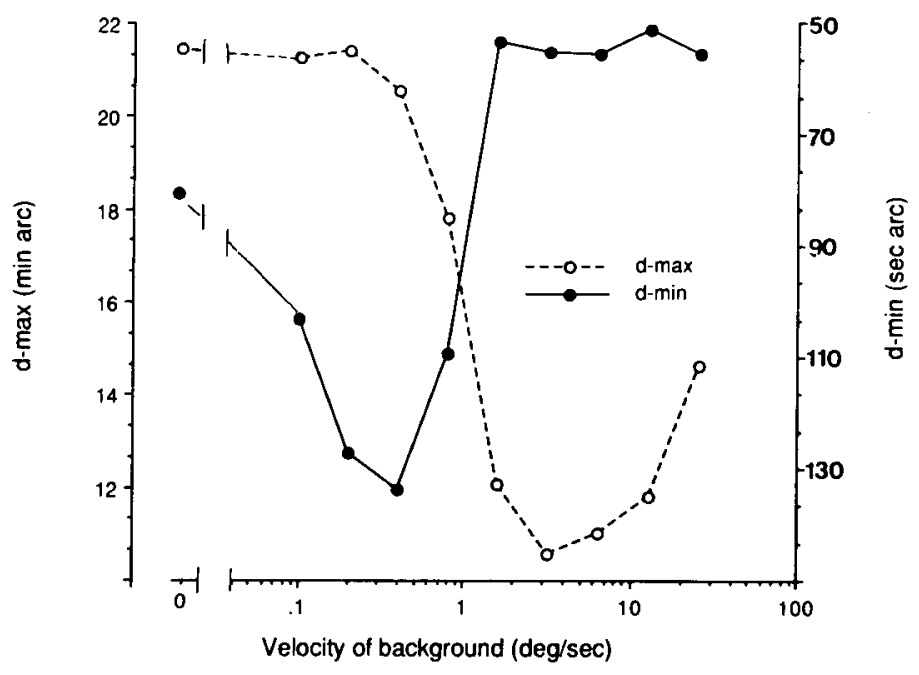

Figure 3. So as to aid comparison of the data from Experiments 1 and 2, the data from 3 subjects in each case has been averaged and replotted. The extreme differences in the shapes of the functions can now clearly be seen. 
dissimilar than is physically the case. Such mutual repulsion has been modeled in other visual dimensions as the product of inhibitory interactions between filters (e.g., see Blakemore, Carpenter, \& Georgeson, 1970). Again, however, the velocities of both patterns have always been the same in these instances, and hence the results do not bear directly upon those of the present study.

On the basis of previous research, it has been suggested that motion perception may involve a cooperative system in which units tuned to similar directions of motion are mutually facilitatory, whereas those tuned to different motions are mutually inhibitory (Chang \& Julesz, 1984; Nawrot \& Sekuler, 1989; Snowden, 1989; Snowden \& Braddick, in press; Williams, Phillips, \& Sekuler, 1986; Williams \& Phillips, 1987). The present experiments were designed to explore the latter type of interaction as a function of speed. The results indicate that these suppressive interactions, although not confined to motions with similar speeds, do not exist between all movements of orthogonal direction. Fast motions (as indicated by $d_{\max }$ ) were only suppressed by motions above a certain velocity, and they were unaffected by slower motions (it is important that this "cutoff velocity" was not simply due to the poorer processing of the lower speeds, for these same speeds gave the most profound effects when $d_{\min }$ was measured). Similarly slow movements (as indicated by $d_{\min }$ ) were only affected by speeds up to a certain cutoff point. The cutoffs between speeds that suppressed $d_{\max }$ and those that did not, and between those that suppressed $d_{\text {min }}$ and those that did not, are quite similar (around $1^{\circ}$ per sec), and they are also quite sharp (see Figure 3). It is tempting to suggest that such a boundary might arise because all slow motions are processed by one network, whereas higher speeds are processed by another. Such divisions between mechanisms of motion detection have been previously drawn (Bonnet, 1982; Boulton, 1987) and may relate in some way to the present results. However, it is also possible to account for the present data in terms of a system of broadly tuned velocity channels.

In conclusion, it appears that suppressive interactions occur between motions in orthogonal directions over a quite large range of velocities. However, the detection of fast motions is unaffected by slow movements, and the detection of slow movements is unaffected by fast movements.

\section{REFERENCES}

ANDERSEN, G. J.(1989). Perception of three-dimensional structure from optic flow without locally smooth velocity. Journal of Experimental Psychology: Human Perception \& Performance, 15, 363-371.

BAKER, C. L., \& BradDick, O. J. (1985a). Eccentricity-dependent scaling of the limits for short-range apparent motion. Vision Research, 25, 803-812.

BaKer, C. L., \& Braddick, O. J. (1985b). Temporal properties of the short-range process in apparent motion. Perception, 14, 181-192.
BARLOW, H. B., \& LEVICK, W. R. (1965). The mechanism of directionally selective units in the rabbit retina. Journal of Physiology, 178, 477-504.

Blakemore, C. B., Carpenter, R. H. S., \& Georgeson, M. A. (1970). Lateral inhibition between orientation detectors in the human visual system. Nature, 228, 37-39.

BonNet, C. (1982). Thresholds of visual motion. In A. H. Wertheim, W. A. Wagenaar, \& H. W. Leibowitz (Eds.), Tutorials on motion perception (pp. 41-79). New York: Plenum.

BoultoN, J. C. (1987). Two mechanisms for the detection of slow motion. Journal of the Optical Society of America A, 4, 1634-1642.

BRADDick, O. J. (1974). A short-range process in apparent motion. Vision Research, 14, 519-527.

Braddick, O. J. (1980). Low-level and high-level processes in apparent motion. Philosophical Transactions of the Royal Society of London, 290B, 137-151.

Chang, J. J., \& Julesz, B. (1984). Cooperative phenomena in apparent motion perception of random-dot cinematograms. Vision Research, 24, 1781-1788.

Clarke, P. G. H. (1977). Subjective standstill caused by interaction of moving patterns. Vision Research, 17, 1243.

HoRn, B. K. P., \& Schunk, B. G. (1981). Determining optical flow. Artificial Intelligence, 17, 185-203.

Marshak, W. M., \&ekuler, R. (1979). Mutual repulsion between moving visual targets. Science, 205, 1399-1401.

Mather, G., \& Moulden, B. (1980). A simultaneous shift in apparent directions: Further evidence for a 'distribution shift' model of direction coding. Quarterly Journal of Experimental Psychology, 32, 325-333.

Nawrot, M., \& SekUler, R. (1989). Assimilation and contrast in motion perception: Explorations in cooperativity. Investigative Ophthalmology \& Visual Science, Supplement 30, 72.

ReICHARDT, W. (1961). Autocorrelation: A principle of the evaluation of sensory information by the central nervous system. In W. A. Rosenblith (Ed.), Sensory communication (pp. 303-317). New York: Wiley.

Siegel, R. M., \& ANDERSEN, R. A. (1988). Perception of threedimensional structure from motion in monkey and man. Nature, 331, 259-261.

SNOWDEN, R. J. (1989). Motions in orthogonal directions are mutually suppressive. Joumal of the Optical Society of America A, 6, 1096-1101.

SNOWden, R. J., \& BRAdDick, O. J. (in press). The combination of motion signals over time. Vision Research.

van Doorn, A. J., KoENDERINK, J. J. (1982a). The spatial properties of the visual detectability of moving white spatial noise. Experimental Brain Research, 45, 189-195.

VAN DOORN, A. J., \& KoEnderunk, J. J. (1982b). The temporal properties of the visual detectability of moving spatial white noise. Experimental Brain Research, 45, 179-188.

VAN DOORN, A. J., Koenderink, J. J. (1982c). Visibility of moving gradients. Biological Cybernetics, 44, 167-175.

WANG, H. T., MATHUR, B., Косн, K. (1989). Computing optical flow in the primate visual system. Neural Computation, 1, 92-103. Wertheimer, M. (1958). Principles of perceptual organization. In D. C. Beardslee (Ed.) \& M. Wertheimer (Trans.), Readings in perception (pp. 115-135). New York: Van Nostrand. (Original work published 1923)

Williams, D., Philuips, G., \& Sekuler, R. (1986). Hysteresis in the perception of motion direction as evidence for neural cooperativity. Nature, 324, 253-255.

Williams, D., Phillips, G. (1987). Cooperative phenomena in the perception of motion direction. Journal of the Optical Society of America A, 4, 878-885.

YUILlE, A. L. \& GRYYACZ, N. M. (1988). A computational theory for the perception of coherent visual motion. Nature, 333, 71-74.

(Manuscript received January 23, 1989; revision accepted for publication August 9, 1989.) 